1 Первый Санкт-Петербургский государственный медицинский университет имени академика И.П. Павлова, Санкт-Петербург, Российская Федерация

${ }^{2}$ Санкт-Петербургский государственный университет, Санкт-Петербург, Российская Федерация

${ }^{3}$ Национальный медицинский исследовательский центр имени В.А. Алмазова, Санкт-Петербург, Российская Федерация

ОБОСНОВАНИЕ. Спонтанное восстановление системы гипофиз-гонады после использования препаратов тестостерона с целью заместительной гормональной терапии или для мужской контрацепции происходит от нескольких месяцев до 2 лет. Недостаточно данных о сроках восстановления системы гипофиз-гонады после злоупотребления андрогенными анаболическими стероидами. Пользователи препаратов тестостерона применяют послекурсовую терапию с целью восстановления системы гипофиз-гонады, эффективность которой неизвестна и требует изучения.

ЦЕЛЬ. Оценить восстановление системы гипофиз-гонады у мужчин-пользователей андрогенных анаболических стероидов на фоне 3-месячного отказа от их введения и применения послекурсовой терапии.

МЕтоды. Проведено обсервационное одноцентровое проспективное выборочное открытое неконтролируемое исследование среди мужчин-пользователей андрогенных анаболических стероидов. На фоне приема препаратов и через 3 мес после отказа от них и проведения послекурсовой терапии учитывали клинические симптомы гипогонадизма, определяли концентрации лютеинизирующего (ЛГ) и фолликулостимулирующего (ФСГ) гормонов, общего тестостерона $\left(\mathrm{T}_{\text {общ }}\right)$, пролактина, эстрадиола, ингибина В, тиреотропного гормона (ТТГ). Схема послекурсовой терапии была одинаковой и не менялась на протяжении исследования. Критерии восстановления: Тоб >3,4 нг/мл и ЛГ >1,24 мМЕ/мл. Исследование проводилось с января по август 2019 г. Использовали точный критерий Фишера, U-критерий Манна-Уитни, коэффициент ранговой корреляции Спирмена. Статистически значимыми считали различия при $p<0,05$.

РЕзУЛЬтАТЫ. Исследование закончили 44 мужчины, средний возраст 29 [27,75; 34] лет, длительность применения андрогенных анаболических стероидов 6 [3,52; 7] мес. На фоне использования стероидов: ЛГ 0,2 [0,04; 0,47] мМЕ/мл, $\mathrm{T}_{\text {общ }} 4,34[1,05 ; 8,81]$ нг/мл. В этой группе было 84\% ( $\left.n=37\right)$ мужчин с уровнем ЛГ <1,24 мМЕ/мл и 47,7\% ( $\left.n=21\right)$ участников с общ $_{\text {об, }}<$ нг/мл. Через 3 мес показатель ЛГ достиг 4,12 [2,58; 5,84] мМЕ/мл, Т общ $-4,55$ [3,76; 6,24] нг/мл, при этом уровень $\mathrm{T}_{\text {общ }}<3,4$ нг/мл остался у 20,5\% ( $\left.n=9\right)$, а ЛГ <1,24 мМЕ/мл - у 4,5\% $(n=2)$ мужчин. По уровню восстановления выработки ЛГ и $\mathrm{T}_{\text {общ }}$ обследованных распределили на две группы: с достаточным $(n=35 ; 79,5 \%)$ и недостаточным ( $\left.n=9 ; 20,5 \%\right)$ восстановлением. Установлена заметная корреляционная связь между длительностью применения $(-0,857 ; p<0,0001)$, количеством $(-0,443 ; p=0,003)$, дозой $(-0,7825 ; p<0,0001)$, видом используемого стероида $(-0,698 ; p<0,0001)$ и восстановлением концентрации $\mathrm{T}_{\text {общ }}$. Выявлена корреляция между ингибином В и $\mathrm{T}_{\text {общ }}(0,418 ; p=0,005)$.

ЗАКЛЮчЕНИЕ. Трехмесячный отказ от использования андрогенных анаболических стероидов на фоне послекурсовой терапии восстановил систему гипофиз-гонады у 79,5\% добровольцев. Установлено отрицательное влияние на восстановление системы гипофиз-гонады вида андрогенных анаболических стероидов, длительности и дозы их использования, а также количества одновременно вводимых препаратов. Уровень ингибина В может служить маркером восстановления сперматогенного эпителия.

КЛЮЧЕВЫЕ СЛОВА: анаболические андрогенные стероиды, злоупотребление стероидами, ингибин В, гипогонадизм.

\title{
PECULIARITY OF RECOVERY OF THE HYPOTHALAMIC-PITUITARY-GONADAL (HPG) AXIS, IN MEN AFTER USING ANDROGENIC ANABOLIC STEROIDS
}

\author{
(c) Mykola P. Lykhonosovi, 2*, Alina Y. Babenko1,3, Viktor A. Makarin², Yuri N. Fedotov
}

\footnotetext{
${ }^{1}$ Pavlov First Saint Petersburg State Medical University, St. Petersburg, Russian Federation 2 University Hospital of Saint-Petersburg State University, St. Petersburg, Russian Federation ${ }^{3}$ Almazov National Medical Research Centre, St. Petersburg, Russian Federation
}

BACKGROUND: Spontaneous recovery of the hypothalamic-pituitary-gonadal (HPG) axis after cessation of testosterone replacement therapy or after male contraception may take up to 24 months. There is insufficient data on the duration of recovery of HPG axis after abuse of androgenic anabolic steroids (AAS). AAS users use post-cycle therapy (PCT) to restore HPG axis, the effectiveness of which is unknown and needs further investigation. 
AIMS: To evaluate the recovery of HPG axis in men, AAS users, after a 3-month of cessation of their use and after PCT.

METHODS: An observational, single-center, prospective, sampling, open, uncontrolled study was conducted among male AAS users. While using of AAS and 3 months after the refusal of their administration and PCT, the clinical symptoms of hypogonadism were evaluated, luteinizing hormone ( $\mathrm{LH})$, follicle-stimulating hormone (FSH), total testosterone (Tt), prolactin, estradiol, inhibin B, thyroid-stimulating hormone (TSH) were determined. The scheme of rehabilitation therapy was the same and did not change throughout the study. Recovery criteria: $\mathrm{Tt}>3.4 \mathrm{ng} / \mathrm{ml}$ and $\mathrm{LH}>1.24 \mathrm{mlU} / \mathrm{ml}$. The study was conducted from January to August 2019. Fisher's exact test, Mann-Whitney U-test, and Spearman's rank correlation coefficient were used. The differences were considered statistically significant at $p<0.05$.

RESULTS: The study included 44 men, their age 29 years [27.75; 34], the duration of the use of AAS is 6 months [3.52; 7]. During the use of AAS: LH $0.2 \mathrm{mlU} / \mathrm{ml}[0.04 ; 0.47], \mathrm{Tt}-4.34 \mathrm{ng} / \mathrm{ml}[1.05 ; 8.81]$. In this group, the number of men with a LH level $<1.24 \mathrm{mIU} / \mathrm{ml}$ was $84 \%(n=37)$ and Tt $<3.4 \mathrm{ng} / \mathrm{ml}$ was $47.7 \%(n=21)$. After 3 months, the LH level reached $4.12 \mathrm{mIU} / \mathrm{ml}[2.58 ; 5.84], \mathrm{Tt}-4.55 \mathrm{ng} / \mathrm{ml}[3.76 ; 6.24]$. At the same time, the level of $\mathrm{Tt}<3.4 \mathrm{ng} / \mathrm{ml}$ remained in $20.5 \%$ ( $n=9$ ), and $\mathrm{LH}<1.24 \mathrm{mlU} / \mathrm{ml}$ in $4.5 \%(n=2)$ men. According to the level of recovery of $\mathrm{LH}$ and Tt, patients were divided into two groups: with satisfying $(n=35 ; 79.5 \%)$ and poor $(n=9 ; 20.5 \%)$ recovery. A clear correlation was established between the duration of use $(-0.857 ; p<0.0001)$, the amount $(-0.443 ; p=0.003)$, the dose $(-0.7825 ; p<0.0001)$, the type of AAS $(-0.698 ; p<0.0001)$ and testosterone level recovery. A correlation between inhibin $B$ and $\mathrm{Tt}(0.418 ; p=0.005)$ was revealed.

CONCLUSIONS: A three-month refusal to use AAS with PCT led to the restoration of HPG axis a in $79.5 \%$ of the volunteers. In $20.5 \%$ of cases, recovery did not occur. The negative effect of the duration of use, the number of simultaneously administered drugs, their dose and type of AAS on the restoration of HPG axis was established. The level of inhibin B may serve as a marker for the restoration of spermatogenic epithelium.

KEYWORDS: anabolic-androgenic steroids, abuse of steroids, behavioral features, hypogonadism, steroid-induced hypogonadism.

\section{ОБОСНОВАНИЕ}

Заместительная терапия тестостероном для лечения возрастного гипогонадизма, а также гипогонадизма, связанного с хроническими заболеваниями, такими как сахарный диабет, ожирение, гипертония и др., может быть причиной подавления системы гипофиз-гонады с развитием снижения фертильности и бесплодия [1]. Применяемые в заместительной терапии тестостероном дозы андрогена и его производных, показания и противопоказания, схемы назначения, длительность, кратность медицинских осмотров и объем лабораторных и инструментальных обследований подробно регламентируются клиническими рекомендациями (протоколами) или национальными руководствами $[2,3]$. Немедицинское использование тестостерона и его производных с целью модификации тела и повышение мышечной силы происходит без учета рекомендуемых фармакологических дозировок и схем и в большинстве случаев наносит существенный вред здоровью пользователей, которые представлены в основном молодыми мужчинами репродуктивного возраста $[4,5]$. Как показали исследования, стероидиндуцированный гипогонадизм (anabolic steroids-induced hypogonadism) оказался одной из часто нераспознаваемых причин развития гипогонадизма у молодых мужчин $[1,6]$. Под влиянием вводимых стероидов снижается выработка гипоталамусом гонадотропин-рилизинг-гормона, что уменьшает продукцию аденогипофизом лютеинизирующего (ЛГ) и фолликулостимулирующего (ФСГ) гормонов. Конверсия на периферии тестостерона ферментом ароматаза в эстроген приводит к угнетению секреции ЛГ и ФСГ. По механизму обратной связи на секрецию ФСГ оказывает влияние и вырабатываемый в яичках ингибин $\mathrm{B}$, считающийся маркером восстановления выработки яичками тестостерона [7]. По литературным данным, после замести- тельной терапии тестостероном или злоупотребления андрогенными анаболическими стероидами спонтанное восстановление системы гипофиз-гонады может продолжаться от нескольких месяцев до 2 лет, при этом описаны случаи необратимого ее повреждения [1]. Пользователи андрогенных анаболических стероидов для снижения негативного влияния препаратов на систему гипофиз-гонады применяют их так называемыми курсами, а между ними проводят послекурсовую терапию (post-cycle therapy), эффективность которой неизвестна, с целью восстановления системы гипофиз-гонады [8]. Таким образом, актуальным является исследование состояния системы гипофиз-гонады при использовании андрогенных анаболических стероидов и через 3 мес послекурсовой терапии на фоне отказа от препаратов тестостерона.

\section{ЦЕЛЬ}

Выявить частоту восстановления системы гипофиз-гонады у мужчин-пользователей андрогенных анаболических стероидов на фоне 3-месячной послекурсовой терапии с исключением препаратов тестостерона.

\section{МЕТОДЫ}

\section{Дизайн исследования}

Проведено обсервационное одноцентровое проспективное выборочное открытое неконтролируемое исследование (рис. 1).

\section{Критерии соответствия}

Критерии включения: возраст от 19 лет и старше, мужской пол, использование андрогенных анаболических стероидов с целью улучшения спортивных показателей и композиции тела, подписание информированного согласия на участие в исследовании. 


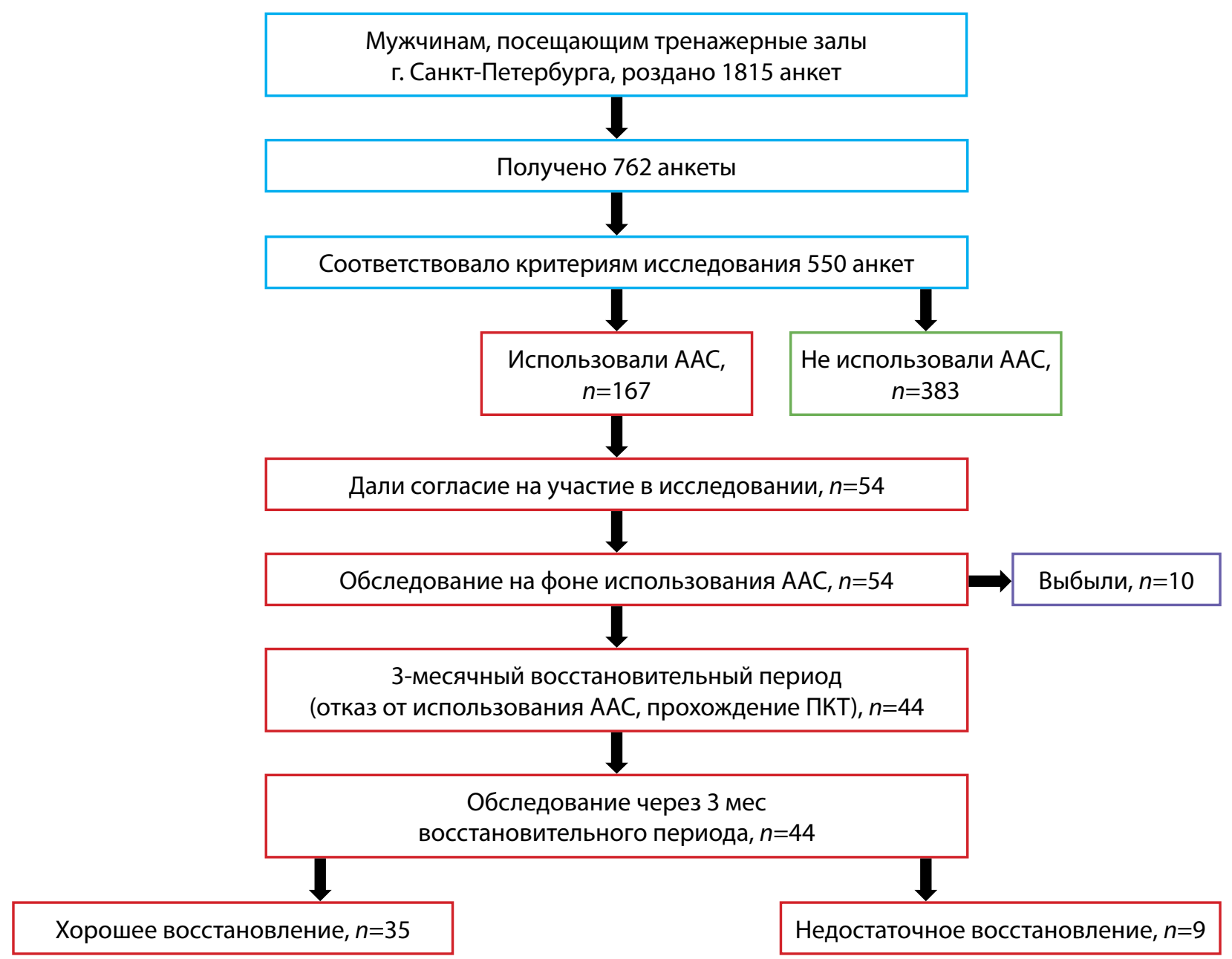

Рис. 1. Дизайн исследования.

Примечание. ААС — андрогенный анаболический стероид; ПКТ — послекурсовая терапия.

Критерии невключения: возраст до 19 лет, занятие профессиональными видами спорта, женский пол.

Критерии исключения: выявление несоответствия критериям включения в ходе исследования, решение отказаться от дальнейшего участия в исследовании.

\section{Условия проведения}

Исследование проведено на базах Первого Санкт-Петербургского государственного медицинского университета имени академика И.П. Павлова и Клиники высоких медицинских технологий им. Н.И. Пирогова Санкт-Петербургского государственного университета.

\section{Продолжительность исследования}

Представляемая работа является вторым, заключительным этапом исследования «Состояние системы гипофиз-гонады у мужчин после использования андрогенных анаболических стероидов», проводимого с ноября 2017 г. по декабрь 2018 г. (первый этап - анкетирование) и с января 2019 г. по август 2019 г. (второй этап - собственно клиническое исследование). На втором этапе после подписания информированного согласия мужчинами-добровольцами, пользователями андрогенных анаболических стероидов, проведены клинико-лабораторные обследования на пике использования стероидов и через 3 мес отказа от их применения на фоне согласованной восстановительной терапии. Смещения запланированных сроков не было.
Описание медицинского вмешательства

Изучали демографические (возраст), анамнестические (длительность использования андрогенных анаболических стероидов, дозы, комбинации), антропометрические (рост, масса тела, рассчитанный индекс массы тела), гемодинамические (артериальное давление) данные. Клинико-лабораторное обследование выполняли согласно Стандартам первичной медико-санитарной помощи при гипогонадизме [9] и проводили дважды: при использовании андрогенных анаболических стероидов и через 3 мес отказа от их применения на фоне согласованной и неизменяемой послекурсовой терапии. Взятие крови для анализов проводилось утром натощак после 8-часового сна, но только до 10 ч утра. В сыворотке крови определяли концентрации ЛГ, ФСГ, общего тестостерона ( $\mathrm{T}_{\text {общ }}$ ), пролактина, эстрадиола, ингибина В, тиреотропного гормона (ТТГ).

Состав послекурсовой терапии у участников исследования соответствовал наиболее используемому у них набору препаратов: кломифена цитрат в дозировке 50 мг/сут в диапазоне от 75 до 90 дней, альфа-токоферола ацетат 100 мг/сут, холекальциферол 5000 ME/нед, цинка сульфат моногидрат по 124 мг 1 раз/сут, L-карнитин по 1,0 г 2 раза/сут. Послекурсовая терапия со всеми включенными в исследование добровольцами предварительно была согласована и не менялась на протяжении 3 мес. 


\section{Исходы исследования}

Основной исход исследования. Выявленная частота восстановления системы гипофиз-гонады после отказа от использования андрогенных анаболических стероидов (критерии восстановления: концентрации $\mathrm{T}_{\text {общ }}$ и ЛГ).

Дополнительные исходы исследования. Вторичными точками в исследовании были концентрации ФСГ, пролактина, эстрадиола, ингибина В, ТТГ, до и через 3 мес после отказа от использования андрогенных анаболических стероидов. Также дополнительно изучалось влияние длительности использования андрогенных анаболических стероидов, их разновидностей, доз, схем применения на восстановление системы гипофиз-гонады.

\section{Анализ в подгруппах}

В исследовании использованы клинико-лабораторные данные 44 мужчин-добровольцев, пользователей андрогенных анаболических стероидов. Оценивали полученные концентрации гормонов в начале (на пике использования стероидов) - массив данных «Исходные значения» и через 3 мес отказа от их применения - массив данных «Через 3 мес». После анализа концентраций ЛГ и $\mathrm{T}_{\text {общ }}$ из массива данных «Через 3 мес» участники были распределены в две подгруппы: «Хорошее восстановление» и «Недостаточное восстановление». Между этими подгруппами выполнен статистический поиск возможных причин различий в восстановлении системы гипофиз-гонады по критерию уровня тестостерона.

\section{Методы регистрации исходов}

Сывороточные концентрации $\mathrm{T}_{\text {общ' }}$ ЛГ, ФСГ, пролактина, эстрадиола определяли иммунохемилюминесцентным методом на анализаторе Access/UniCel Dxl Beckman Coulter (США). Референсные значения: тестостерон для мужчин в возрасте от 18 лет 1 дня до 67 лет 1,75-7,81 нг/мл; ЛГ для мужчин от 19 лет 1 дня до 120 лет 1,24-8,62 мМЕ/мл; ФСГ для мужчин от 19 лет 1 дня до 120 лет 1,27-19,26 мМЕ/мл; пролактин для мужчин от 18 лет 1 дня до 120 лет 55,97-278,36 мМЕ/мл; эстрадиол для мужчин от 19 лет 1 дня до 120 лет 73,42-172,54 пмоль/л.

Концентрации ингибина В определяли иммуноферментным методом на анализаторе Infinite F50 Tecan (GmbH, Австрия); HydroFlex V 8/2 Tecan (GmbH, Австрия). Референсные значения для мужчин от 14 лет 1 дня до 120 лет 25,0-325,0 пг/мл.

Концентрации ТTГ определяли иммунохемилюминесцентным методом на анализаторе ARCHITECT i2000SR (Abbott Laboratories, США). Референсные значения для мужчин от 15 лет 1 дня до 21 года 0,43-4,2 мкMЕ/мл, а с 21 года 1 дня до 120 лет 0,35-4,94 мкMЕ/мл.

Полученные образцы крови сразу направлялись для лабораторной диагностики согласно протоколам лабораторного исследования. Хранение крови не предусматривалось. Концентрации ЛГ, ФСГ, Т общ определялись в одном образце крови.

Критериями восстановления системы гипофиз-гонады после отмены андрогенных анаболических стероидов и послекурсовой терапии были концентрации $\mathrm{T}_{\text {общ }}>3,4$ нг/мл ( $\geq 12,0$ нмоль/л) и ЛГ >1,24 мМЕ/мл $[3,9]$.

\section{Этическая экспертиза}

Этический комитет ФГБОУ ВО «Первый Санкт-Петербургский государственный медицинский университет им. акад. И.П. Павлова» Минздрава России постановил одобрить проведение исследования «Состояние системы гипофиз-гонады у мужчин после использования андрогенных анаболических стероидов» (Протокол № 8 от 24 ноября 2017). Все пациенты добровольно подписали информированное согласие на участие в исследовании.

\section{Статистический анализ}

Расчет объема выборки проведен по формуле (M. Bland, 2000):

\section{$n=15,4 \times p \times(1-p) / W^{2}$,}

где $n$ - необходимый размер выборки; $p$ - ожидаемая частота результата (5\%); (100 - p) - частота обратного события (95\%); W - ширина доверительного интервала (20\%). В группе должно быть до 20 человек $(15,4 \times 5 \times 95 / 400=19)$. С учетом отклика выборку увеличили на 20\% (4 человека), и скорректированный размер выборки составил не менее 24 участников [10]. Все этапы исследования закончили 44 человека, их данные легли в основу статистического анализа.

Использован лицензионный статистический пакет SAS 9.4 (SAS Institute Inc., SAS Campus Drive, Кэри, Северная Каролина, США). Данные представлены в виде медианы и интерквартильного размаха - Mediana [Q25; Q75]. Применяли Т-критерий Вилкоксона для сравнения показателей в группах до и после исследования, U-критерий Манна-Уитни для количественных данных, точный критерий Фишера для качественных показателей, коэффициент ранговой корреляции Спирмена для оценки взаимосвязи между показателями. Статистически значимыми считали различия при $p<0,05$.

\section{РЕЗУЛЬТАТЫ}

\section{Объекты (участники) исследования}

Изъявили желание участвовать в исследовании 105 мужчин, добровольно заявивших, что используют андрогенные анаболические стероиды. После индивидуального собеседования, объяснения целей и протокола исследования информированное согласие подписали только 54 человека (51,4\% от изначально желающих быть включенным в исследование). По итогам первого клинического осмотра выбыли 10 (9,5\%) человек. Все этапы исследования прошли 44 добровольца (41,9\%): мужчины, занимающиеся физической рекреационной активностью, согласившиеся с условиями исследования и указавшие на использование андрогенных анаболических стероидов. Исходная характеристика обследованных мужчин-добровольцев $(n=44)$, пользователей андрогенных анаболических стероидов, представлена в табл. 1.

Таким образом, все участники - это мужчины оптимального репродуктивного возраста, хорошо образованные, с продолжительностью введения стероидов от 6 до 9 мес в дозе 1000 мг и выше, чаще использующие одновременно 2 и более андрогенных анаболических стероида, комбинируя тестостерона пропионат с производными дигидротестостерона и/или 19-нортестостерона. 
Таблица 1. Общая характеристика участников-добровольцев исследования

\begin{tabular}{|c|c|}
\hline Параметр & $\begin{array}{c}\text { Пользователи АAC } \\
n=44\end{array}$ \\
\hline Мужчины, абс. (\%) & $44(100)$ \\
\hline Возраст, лет & $29[27,7 ; 34]$ \\
\hline \multicolumn{2}{|c|}{ Возрастные группы, лет } \\
\hline $18-21$ & $2(4,6)$ \\
\hline $22-35$ & $35(79,5)$ \\
\hline $36-45$ & $6(13,6)$ \\
\hline $46-60$ & $1(2,3)$ \\
\hline Индекс массы тела, кг/м² & $29,5[28 ; 33]$ \\
\hline \multicolumn{2}{|c|}{ Образование } \\
\hline Среднее & $12(27)$ \\
\hline Высшее & $32(73)$ \\
\hline Использование андрогенных анаболических стероидов & Да, 44 (100) \\
\hline \multicolumn{2}{|c|}{ Продолжительность использования ААС, мес } \\
\hline $3-5$ & $4(9)$ \\
\hline $6-9$ & $29(66)$ \\
\hline Более 9 & $11(25)$ \\
\hline \multicolumn{2}{|c|}{ Дозы инъекционных препаратов ААС, мг } \\
\hline До 750 & $11(25)$ \\
\hline До 1000 & $16(36)$ \\
\hline Более 1000 & $17(39)$ \\
\hline \multicolumn{2}{|c|}{ Количество одновременно используемых видов ААС, фарм. препарат } \\
\hline 1 & $8(18)$ \\
\hline 2 & $22(50)$ \\
\hline 3 & $14(32)$ \\
\hline \multicolumn{2}{|c|}{ Виды АAC } \\
\hline Тестостерона пропионат & $43(97,7)$ \\
\hline Производные дигидротестостерона & $29(65,9)$ \\
\hline Производные 19-нортестостерона & $21(47,7)$ \\
\hline Использование послекурсовой терапии & $44(100)$ \\
\hline Прием тиреоидных препаратов & $2(4,5)$ \\
\hline
\end{tabular}

Примечание. Данные представлены в виде Ме [Q25; Q75]. АAC — андрогенные анаболические стероиды.

Использовали тиреоидные препараты в целях модификации тела двое участников, их данные по ТТГ были исключены из статистического анализа. На клинические проявления гипогонадизма (снижение либидо, эректильная дисфункция) указали только 4,5\% $(n=2)$ мужчин.

\section{Основные результаты исследования}

Все 44 добровольца прошли лабораторное обследование на фоне использования андрогенных анаболических стероидов и спустя 3 мес их исключения из рациона при одновременном проведении послекурсовой терапии (табл. 2).

При сравнении показателей $\mathrm{T}_{\text {общ }}$ у участников исследования при использовании андрогенных анаболических стероидов и через 3 мес после их исключения на фоне послекурсовой терапии достоверной разницы не выявлено ( $p=0,801)$, однако число мужчин с уровнем $\mathrm{T}_{\text {общ }}<3,4$ нг/мл через $3 \mathrm{mес} \mathrm{исследования} \mathrm{достоверно}$ снизилось $(p=0,004)$.
Показатели ЛГ и ФСГ на фоне применения андрогенных анаболических стероидов определялись значительно ниже нормы, но через 3 мес послекурсовой терапии с полным исключением препаратов тестостерона уже соответствовали нормальным значениям (для обоих гормонов $p<0,001)$.

Не выявлено отличий между начальными и кончеными показателями пролактина и эстрадиола ( $p=0,543$ и $p=0,248$ соответственно).

Сравнение исходных и конечных показателей ингибина В выявило достоверное увеличение этого гормона в сыворотке добровольцев группы «Через 3 мес» $(p<0,001)$, при этом содержание ТТГ у них также значимо увеличилось, но осталось в диапазоне нормальных значений $(p=0,010)$.

По лабораторным критериям диагностики гипогонадизма гипогонадотропный гипогонадизм (ЛГ <1,24 мМЕ/мл и $\mathrm{T}_{\text {общ }}<3,4$ нг/мл) был выявлен у 47,7\% ( $\left.n=21\right)$ мужчин в начале исследования. Нами установлено, что при концен- 
Таблица 2. Лабораторные показатели участников-добровольцев в условиях проводимого исследования

\begin{tabular}{|c|c|c|c|}
\hline Показатель & $\begin{array}{c}\text { Исходные данные } \\
\text { (на фоне АAC) }\end{array}$ & $\begin{array}{c}\text { Через } 3 \text { мес } \\
\text { (6ез AAC с ПКТ) }\end{array}$ & $p$ \\
\hline $\begin{array}{l}\mathrm{T}_{\text {общ' }} \text { нг/мл; абс. (\%) } \\
<3,4 \\
\geq 3,4\end{array}$ & $\begin{array}{c}4,34[1,3 ; 8,81] \\
21(47,7) \\
23(52,3)\end{array}$ & $\begin{array}{c}4,55[3,8 ; 6,2] \\
9(20,5) \\
35(79,5)\end{array}$ & $\begin{array}{l}p^{*}=0,801 \\
x^{2}=8,692 \\
p=0,004\end{array}$ \\
\hline $\begin{array}{l}Л Г, \text { мМЕ/мл } \\
<1,24 \\
\geq 1,24\end{array}$ & $\begin{array}{c}0,2[0,04 ; 0,5] \\
37(84) \\
7(16)\end{array}$ & $\begin{array}{c}4,11[2,6 ; 5,8] \\
2(4,5) \\
42(95,5)\end{array}$ & $\begin{array}{l}p^{*}<0,001 \\
X^{2}=56,92 \\
p<0,001\end{array}$ \\
\hline $\begin{array}{l}\text { ФСГ, мМЕ/мл } \\
<1,27 \\
\geq 1,27\end{array}$ & $\begin{array}{c}0,35[0,2 ; 0,6] \\
41(93) \\
3(7)\end{array}$ & $\begin{array}{c}4,36[2,4 ; 5,2] \\
2(4,5) \\
42(95,5)\end{array}$ & $\begin{array}{l}p^{*}<0,001 \\
X^{2}=69,17 \\
p<0,001\end{array}$ \\
\hline Пролактин, мМЕ/мл & $145,1[113,4 ; 201,4]$ & $140,8[122,2 ; 158,4]$ & $p^{*}=0,543$ \\
\hline Эстрадиол, пмоль/л & $134,3[73 ; 173,9]$ & $102,6[79,2 ; 150,8]$ & $p^{*}=0,248$ \\
\hline Ингибин В, пг/мл & $131,7[72,3 ; 166,4]$ & $186,8[142,3 ; 228,5]$ & $p^{*}<0,001$ \\
\hline ТТГ, мкМЕ/мл & $1,14[0,9 ; 1,76]$ & $1,29[1 ; 2,2]$ & $p^{*}=0,010$ \\
\hline
\end{tabular}

Примечание. Данные представлены в виде Мe [Q25; Q75]. $p$ - уровень значимости по критерию X2 для произвольных таблиц; - уровень значимости по критерию Вилкоксона для парных сравнений. АAC - андрогенные анаболические стероиды; ПКТ — послекурсовая терапия; $\mathrm{T}_{\text {общ }}$ - тестостерон общий; ЛГ — лютеинизирующий гормон; ФСГ — фолликулостимулирующий гормон; ТТГ - тиреотропный гормон.

трации ЛГ <1,24 мМЕ/мл у 22,7\% ( $n=10)$ участников $\mathrm{T}_{\text {общ }}$ был в нормальных границах — от 3,4 до 7,81 нг/мл (Mе $5,6[5,2 ; 7,2]$ нг/мл), а у $29,5 \%$ ( $n=13)$ участников показатель $\mathrm{T}_{\text {общ }}$ был существенно выше нормальных значений (Ме 10,57 [8,92; 30,79] нг/мл). Неестественно высокое содержание $\mathrm{T}_{\text {общ }}$ при почти нулевых значениях ЛГ и ФСГ объясняется тем, что уровень определяемого в крови $\mathrm{T}_{\text {общ }}$ не является истинным (собственным), а обусловлен вводимым препаратом Testosterone propionate. Именно поэтому количество мужчин с гипогонадотропным гипогонадизмом следует учитывать по ЛГ <1,24мME/мл, который отмечался у $84 \%(n=37)$ мужчин. У остальных $16 \%(n=7)$ обследованных добровольцев Лг был выше 1,24 мМЕ/мл, вероятно, вследствие того, что система гипофиз-гонады не успела отреагировать на использование андрогенных анаболических стероидов.

Через 3 мес полного отказа от использования андрогенных анаболических стероидов и на фоне послекурсовой терапии мужчин с уровнем ЛГ <1,24 мМЕ/мл осталось $4,5 \%(n=2)$, а с Т общ $<3,4$ нг/мл - 20,5\% (n=9). По лабораторным показателям, гипогонадотропный гипогона- дизм остался у 20,5\% ( $n=9)$ участников исследования. У 79,5\% ( $n=35)$ обследованных, согласно лабораторным показателям, система гипофиз-гонады восстановилась: ЛГ > 1,24 мМЕ/мл, общ $_{\text {оз }} \geq 3,4$ нг/мл.

В то же время число мужчин, указавших на клинические проявления гипогонадизма спустя 3 мес после исключения андрогенных анаболических стероидов из рациона, достигло 29,5\% ( $n=13)$. Таким образом, сопоставление лабораторных показателей с клинической симптоматикой продемонстрировало восстановление системы гипофиз-гонады в 70,5\% случаев $(n=31)$ и отсутствие восстановления в $29,5 \%(n=13)$.

Дополнительные результаты исследования

Для дальнейших расчетов основных критериев восстановления системы гипофиз-гонады в этом исследовании использовали показатели $\mathrm{T}_{\text {общ }} \geq 3,4$ нг/мл. Были сформированы подгруппы «Хорошее восстановление» ( $n=35)$ и «Недостаточное восстановление» $(n=9)$. Результаты сравнения данных подгрупп представлены в табл. 3.

Таблица 3. Сравнение параметров подгрупп участников-добровольцев с хорошим и недостаточным восстановлением системы гипофиз-гонады (критерий восстановления: уровень $\mathrm{T}_{\text {общ }} \geq 3,4 \mathrm{Hr} / \mathrm{Mл)}$

\begin{tabular}{lccc}
\hline \multicolumn{1}{c}{ Параметр } & $\begin{array}{c}\text { Хорошее восстановление } \\
\mathbf{T}_{\text {общ }}<\mathbf{3 , 4} \text { нг/мл }\end{array}$ & $\begin{array}{c}\text { Недостаточное } \\
\text { восстановление } \\
\mathbf{T}_{\text {общ }} \geq \mathbf{3 , 4} \mathbf{4 r} / \mathbf{M л}\end{array}$ & $\boldsymbol{p}^{*}$ \\
\hline Возраст, лет & $29[27 ; 32,5]$ & $31[28,8 ; 35,8]$ & 0,186 \\
Индекс массы тела, кг/м² & $29[28 ; 32,5]$ & $33[28,7 ; 34,3]$ & 0,343 \\
ЛГ, мМЕ/мл & $0,19[0,03 ; 0,3]$ & $0,21[0,1 ; 1,2]$ & 0,194 \\
ФСГ, мМЕ/мл & $0,28[0,2 ; 0,5]$ & $0,81[0,2 ; 0,9]$ & 0,439 \\
Пролактин, мМЕ/мл & $136,55[85,5 ; 166,2]$ & $231,9[181,4 ; 243,7]$ & 0,006 \\
Эстрадиол, пмоль/л & $130,4[73 ; 160,7]$ & $86,3[73 ; 144,6]$ & 0,606 \\
Ингибин В, пг/мл & $127,4[79 ; 152,4]$ & $106[80 ; 185,8]$ & 0,954 \\
ТТГ, мкМЕ/мл & $1,04[0,8 ; 1,6]$ & $1,39[1,2 ; 2]$ & 0,328 \\
\hline
\end{tabular}

Примечание. Данные представлены в виде Ме [Q25; Q75]. * — уровень значимости по U-критерию Манна-Уитни. Т общ - тестостерон общий, лг — лютеинизирующий гормон, ФСГ — фолликулостимулирующий гормон, ТТГ — тиреотропный гормон. 
В нашем исследовании между подгруппами хорошего и недостаточного восстановления отличий по возрасту, индексу массы тела, начальным показателям ЛГ, ФСГ, эстрадиола, ингибина В и ТТГ не выявлено. Значимое отличие выявлено только по содержанию пролактина: чем выше была его концентрация в начале исследования, тем хуже было восстановление секреции тестостерона $(p=0,006)$.

Корреляционный анализ выявил заметные отрицательные связи между длительностью использования андрогенных анаболических стероидов $(r=-0,855 ; p<0,0001)$, количеством используемых препаратов $(r=-0,857 ; p<0,0001)$, а также умеренные положительные связи между начальными уровнями ЛГ $(r=0,330 ; p=0,029)$, ингибина $\mathrm{B}(r=0,418$; $p=0,005)$ и восстановлением системы гипофиз-гонады, определяемым по концентрации $\mathrm{T}_{\text {общ. }}$

Нежелательные явления

Не отмечались.

\section{ОБСУЖДЕНИЕ}

\section{Резюме основного результата исследования}

Использование андрогенных анаболических стероидов с целью улучшения композиции тела и повышения мышечной силы осуществляется с выраженным нарушением режима дозирования, что приводит к значительному угнетению системы гипофиз-гонады. В нашем исследовании на фоне использования стероидов концентрации ЛГ и ФСГ были ниже нижней границы нормы у 4 из 5 обследованных, клинические симптомы гипогонадизма присутствовали менее чем у 5\% мужчин. При этом $\mathrm{T}_{\text {общ }}$ в половине случаев имел значения выше или равные 3,4 нг/мл, что объясняется циркуляцией ранее введенного Testosterone propionate. Через 3 мес полного исключения андрогенных анаболических стероидов на фоне послекурсовой терапии концентрации ЛГ и ФСГ восстановились в подавляющем числе случаев; система гипофиз-гонады по лабораторным показателям (уровни ЛГ > 1,24 мМЕ/мл и $\mathrm{T}_{\text {общ }} \geq 3,4$ нг/мл) восстановилась у 79,5\% мужчин.

\section{Обсуждение основного результата исследования}

Согласно определению, «гипогонадизм у мужчин это клинический и биохимический синдром, связанный с низким уровнем тестостерона, а также нечувствительностью рецепторного аппарата к андрогенам, который может оказывать негативное воздействие на множество органов и систем, ухудшая качество жизни и жизненный прогноз» [2]. В нашем исследовании лишь 4,5\% $(n=2)$ мужчин можно было диагностировать гипогонадизм на фоне использования андрогенных анаболических стероидов с целью улучшения формы тела и увеличения мышечной силы. Как оказалось, клиническая симптоматика гипогонадизма (снижение либидо, эректильная дисфункция) нехарактерна для периода активного использования андрогенных анаболических стероидов. В то же время у большинства пользователей андрогенных анаболических стероидов показатели ЛГ, ФСГ и частично $\mathrm{T}_{\text {общ }}$ оказались значительно ниже нижней границы нормальных показателей. При этом уровень $\mathrm{T}_{\text {общ }}$ у половины обследованных соответствовал нормальным значениям или был выше верхней границы нормы. В нашем иссле-

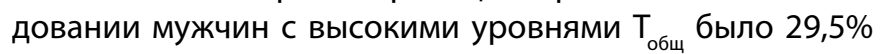
$(n=13)$, а максимальное значение $\mathrm{T}_{\text {общ }}$ соответствовало 45,56 нг/мл (157,96 нмоль/л). Считается, что нормальный уровень тестостерона в сыворотке крови взрослых мужчин соответствует 3-10 нг/мл [8]. Правила сдачи анализа крови для определения концентраций тестостерона, ЛГ и ФСГ предусматривали отказ от введения в течение 48 ч андрогенных анаболических стероидов, что оговаривалось на этапе включения в исследование и непосредственно при выдаче направлений на анализы. Однако проверить выполнение всех оговоренных условий в реальности не представлялось возможным. В этой связи в качестве диагностического критерия ответа системы гипофиз-гонады на вводимые андрогенные анаболические стероиды следует считать концентрацию ЛГ <1,24 мМЕ/мл. Во время полного отказа от стероидов (в нашем случае этот период длился 3 мес) концентрация экзогенного тестостерона снижается, и при восстановлении выработки ЛГ, воздействующего на клетки Лейдига, вырабатывается собственный тестостерон. Поэтому после 3-месячного отказа от использования стероидов критериями восстановления системы гипофиз-гонады могут служить ЛГ $\geq 1,24$ мME/мл и $\mathrm{T}_{\text {общ }} \geq 3,4$ нг/мл. Случаев, удовлетворяющих этим лабораторным параметрам, в нашем исследовании было 79,5\%. Однако мужчин, у которых одновременно не было выявлено ни клинических, ни лабораторных признаков гипогонадизма, оказалось 70,5\%. Обращает на себя внимание значимо увеличившийся после отмены андрогенных анаболических стероидов процент мужчин (с 4,5 до 29,5\%), у которых снизилось качество жизни, появились клинические жалобы на либидо, эректильную дисфункцию. Такое ухудшение в самочувствии пользователей андрогенных анаболических стероидов частично объясняет высокую частоту рецидива использования стероидов [11].

Сложившаяся парадигма использования андрогенных анаболических стероидов у мужчин, занимающихся рекреационной физической деятельностью, не позволила нам набрать контрольную группу, участники которой должны были отказаться от использования стероидов и не получать послекурсовой терапии, поэтому все обсуждаемые результаты исследования получены нами на фоне послекурсовой терапии. Литературные источники указывают на разнообразный набор лекарственных препаратов, используемых в послекурсовой терапии $[8,12,13]$. В нашем исследовании состав препаратов для послекурсовой терапии, их дозы, длительность применения соответствовали наиболее распространенным среди пользователей андрогенных анаболических стероидов в городе Санкт-Петербурге [5], однако применяемую схему послекурсовой терапии нельзя считать как обеспечившей достигнутые результаты по восстановлению системы гипофиз-гонады вследствие отсутствия группы сравнения, включающей мужчин соответствующего возраста, также отказавшихся от использования андрогенных анаболических стероидов, но не принимавших послекурсовой терапии. Эффективность применения послекурсовой терапии требует дальнейшего изучения.

Известно, что выработка клетками Лейдига тестостерона регулируется ЛГ и опосредованно эстрадиолом, пролактином и ингибином В [1]. Мы не обнаружили 
влияния уровней эстрадиола и пролактина на восстановление выработки тестостерона, что обусловлено, возможно, недостаточной для этих целей выборкой. Но связь между степенью угнетения системы гипофиз-гонады и снижением выработкой тестостерона, определяемая по уровню ингибина В, вырабатываемого яичками, подтверждена. Концентрации ингибина В значимо коррелировали с уровнями $\mathrm{T}_{\text {общ }}(p=0,005)$, что подтверждает возможность применения показателя ингибина В как маркера восстановления сперматогенеза [14].

В нашем исследовании выявлено статистически значимое отрицательное влияние на восстановление выработки тестостерона таких факторов, как доза вводимого стероида ( $p=0,0032)$, количество одновременно используемых препаратов $(p<0,0001)$, прием производных дигидротестостерона $(p=0,0181)$ и 19-нортестостерона ( $p=0,0005)$ : чем выше вводимая доза, чем большее количество препаратов, в том числе производных дигидротестостерона и 19-нортестостерона, использовалось, тем реже наступало восстановление системы гипофизгонады.

Известно, что стероиды влияют на функцию щитовидной железы, активируя через глюкокортикоидный рецептор ингибирование синтеза и секреции ТТГ, и снижают содержание тироксинсвязывающего глобулина [15]. Сравнение показателей ТТГ на фоне приема андрогенных анаболических стероидов и через 3 мес отказа от их использования подтвердило значимо более низкий результат содержания гормона при введении препаратов тестостерона.

\section{Ограничения исследования}

Ограничениями считались препараты андрогенных анаболических стероидов, их дозы, частота и длительность использования, заявленные участниками исследования, но не поддающиеся проверке; также отсутствовал механизм контроля выполнения послекурсовой терапии: все основывалось на честности и заинтересованности добровольцев в результатах медицинского обследования. Желающих отказаться от использования андрогенных анаболических стероидов без восстановительного лечения и составить группу контроля не оказалось. Полученные результаты относятся только к мужчинам, занимающимся рекреационной физической активностью.

\section{ЗАКЛЮЧЕНИЕ}

Злоупотребление андрогенными анаболическими стероидами для улучшения композиции тела и повышения мышечной силы остается широко распростра- ненным явлением, несмотря на доступную информацию о нежелательных явлениях и осложнениях в виде снижения либидо и фертильности. Эта информация часто игнорируется основным контингентом пользователей андрогенных анаболических стероидов, представленным молодыми мужчинами репродуктивного возраста. С целью профилактики осложнений у приверженцев препаратов тестостерона выработана парадигма использования андрогенных анаболических стероидов, предполагающая их введение так называемыми курсами с последующей курсовой терапией, эффективность которой остается пока неясной. Неизученными остаются и сроки самостоятельного восстановления системы гипофиз-гонады после использования андрогенных анаболических стероидов. В нашем исследовании выявлено значительное угнетение выработки ЛГ и ФСГ у подавляющего числа обследованных участников на фоне применения андрогенных анаболических стероидов. Восстановление системы гипофиз-гонады отмечалось не у всех мужчин: каждый пятый участник после отказа от использования стероидов на фоне 3-месячной послекурсовой терапии отмечал снижение либидо и симптоматику эректильной дисфункции, что указывает на высокие риски угрозы здоровью мужчин при злоупотреблении андрогенными анаболическими стероидами, даже с учетом общепринятых схем использования и проведения послекурсовой терапии.

\section{ДОПОЛНИТЕЛЬНАЯ ИНФОРМАЦИЯ}

Источник финансирования. Исследование выполнено при финансовой поддержке Клиники высоких медицинских технологий им. Н.И. Пирогова Санкт-Петербургского государственного университета.

Конфликт интересов. Авторы декларируют отсутствие явных и потенциальных конфликтов интересов, связанных с публикацией настоящей статьи.

Участие авторов. Дизайн исследования, поиск литературы, разработка и оформление анкет, опросников, работа с участниками исследования, клинический осмотр, формирование групп, оформление медицинской документации, обеспечение прохождения лабораторного обследования, курация участников на всем протяжении исследования, получение и обработка лабораторных заключений, анализ массива полученных данных, написание текста статьи, формулирование выводов - Н.П. Лихоносов; концепция и научное руководство проектом, коррекция текста статьи, помощь в интерпретации полученных данных - А.Ю. Бабенко; работа с участниками исследования, поиск литературных данных - В.А. Макарьин, помощь в интерпретации полученных данных, коррекция текста статьи - Ю.Н. Федотов. Все авторы внесли значимый вклад в проведение исследования и подготовку статьи, прочли и одобрили финальную версию статьи перед публикацией.

\section{СПИСОК ЛИТЕРАТУРЫ | REFERENCES}

1. McBride JA, Coward RM. Recovery of spermatogenesis following testosterone replacement therapy or anabolic androgenic steroid use. Asian J Androl. 2016;18(3):373-380 doi: https://doi.org/10.4103/1008-682x.173938.

2. Дедов И.И., Мельниченко Г.А., Роживанов Р.В., Курбатов Д.Г. Рекомендации по диагностике и лечению гипогонадизма (дефицита тестостерона) у мужчин. Проект // Проблемы эндокринологии. - 2015. - Т.61. — №5. - C. 60-71. [Dedov II,
Mel'nichenko GA, Rozhivanov RV, Kurbatov DG. The recommendations on diagnostics and treatment of male hypogonadism (deficit of testosterone). The project. Problems of Endocrinology. 2015;61(5):60-71. (In Russ).] doi: https://doi.org/10.14341/probl201561560-71.

3. Bhasin S, Brito JP, Cunningham GR, et al. Testosterone therapy in men with hypogonadism: an Endocrine Society Clinical Practice Guideline. J Clin Endocrinol Metab. 2018;103(5):1715-1744. doi: https://doi.org/10.1210/jc.2018-00229. 
4. El Osta R, Almont T, Diligent C, et al. Anabolic steroids abuse and male infertility. Basic Clin Androl. 2016;26:2. doi: https://doi.org/10.1186/s12610-016-0029-4.

5. Лихоносов Н.П., Бабенко А.Ю. Медицинский аспект использования анаболических андрогенных стероидов у мужчин, посещающих тренажерные залы Санкт-Петербурга // Проблемы эндокринологии. — 2019. — Т.65. — №1. —C. 19-30. [Lykhonosov MP, Babenko AY. The medical aspect of using anabolic androgenic steroids in males attending gyms of SaintPetersburg. Problems of Endocrinology. 2019;65(1):19-30. (In Russ).] doi: https://doi.org/10.14341/probl9832.

6. Park HJ. Anabolic steroid-induced hypogonadism: a challenge for clinicians. J Exerc Rehabil. 2018;14(1):2-3. doi: https://doi.org/10.12965/jer.1836036.018.

7. Zhu ZG, Zhao ZG, Pang QY, et al. Predictive significance of serum inhibin B on testicular haploid gamete retrieval outcomes in nonobstructive azoospermic men. Asian J Androl. 2019;21 (2):137-142. doi: https://doi.org/10.4103/aja.aja_94_18.

8. Tatem AJ, Beilan J, Kovac JR, Lipshultz LI. Management of anabolic steroid-induced infertility: novel strategies for fertility maintenance and recovery. World J Mens Health. 2020;38(2):141-150 doi: https://doi.org/10.5534/wjmh.190002.

9. Приказ Минздрава России от 09.11.2012 № 780н «Об утверждении стандарта первичной медико-санитарной помощи при гипогонадизме». [Order of the Ministry of Health of the Russian federation № 780n «Ob utverzhdenii standarta pervichnoi medikosanitarnoi pomoshchi pri gipogonadizme» dated 09.11.2012. (In Russ).] Доступно по: http://base.garant.ru/70304310/. Ссылка активна на 12.02.2020.

10. Bland M. An introduction to medical statistics. 3rd. ed. Oxford: Oxford University Press; 2000. P. 335-347.

11. Collins R. The war on anabolic-androgenic steroids. In: Van de Ven K, Mulrooney KJ, McVeigh J, editors. Human enhancement drugs. London: Routledge; 2019. P. 264-279. doi: https://doi.org/10.4324/9781315148328-19.

12. Surampudi P, Swerdloff RS, Wang C. An update on male hypogonadism therapy. Expert Opin Pharmacother. 2014;15(9):1247-1264. doi: https://doi.org/10.1517/14656566.2014.913022

13. Christou MA, Tigas S. Recovery of reproductive function following androgen abuse. Curr Opin Endocrinol Diabetes Obes. 2018;25(3):195-200. doi: https://doi.org/10.1097/med.0000000000000406.

14. Kumanov P, Nandipati K, Tomova A, Agarwal A. Inhibin B is a better marker of spermatogenesis than other hormones in the evaluation of male factor infertility. Fertil Steril. 2006;86(2):332-338. doi: https://doi.org/10.1016/j.fertnstert.2006.01.022.

15. Haugen BR. Drugs that suppress TSH or cause central hypothyroidism. Best Pract Res Clin Endocrinol Metab. 2009;23(6):793-800. doi: https://doi.org/10.1016/j.beem.2009.08.003.

\section{ИНФОРМАЦИЯ ОБ АВТОРАХ [AUTHORS INFO]}

*Лихоносов Николай Павлович, аспирант [Mykola P. Lykhonosov, MD, PhD-student]; адрес: 197022, Санкт-Петербург, ул. Льва Толстого, д. 6-8 [address: 6/8 L'va Tolstogo street, 197022 St. Petersburg, Russia]; e-mail: likhonosov.pn@gmail.com, SPIN-код: 2102-5358, ORCID: https://orcid.org/0000-0001-6460-5226

Бабенко Алина Юрьевна, д.м.н., доцент [Alina Y. Babenko, MD, PhD, Associate Professor]; e-mail: alina_babenko@mail.ru, SPIN-код: 9388-1077, ORCID: https://orcid.org/0000-0002-0559-697X

Макарьин Виктор Алексеевич, к.М.н. [Viktor A. Makarin, MD, PhD]; e-mail: mvaviktor@mail.ru, SPIN-код: 8885-2085, ORCID: https://orcid.org/0000-0003-1710-8045

Федотов Юрий Николаевич, д.м.н., професcop [Yury N. Fedotov, MD, PhD, Professor]; e-mail: 6762525@gosmed.ru, ORCID: https://orcid.org/0000-0003-0445-9428

\section{ЦИТИРОВАТЬ:}

Лихоносов Н.П., Бабенко А.Ю., Макарьин В.А., Федотов Ю.Н. Особенности восстановления системы гипофиз-гонады у мужчин после использования андрогенных анаболических стероидов // Проблемы эндокринологии. - 2020. T.66. — №1. — С. 104-112. doi: https://doi.org/10.14341/probl12223

\section{TO CITE THIS ARTICLE:}

Lykhonosov MP, Babenko AY, Makarin VA, Fedotov YN. Peculiarity of Recovery of the Hypothalamic-PituitaryGonadal (HPG) Axis, in Men After Using Androgenic Anabolic Steroids. Problems of Endocrinology. 2020;66(1):104-112. doi: https://doi.org/10.14341/probl12223 